\title{
Multistability in the Kuramoto model with synaptic plasticity
}

\author{
Yuri L. Maistrenko, ${ }^{1,2}$ Borys Lysyansky, ${ }^{1}$ Christian Hauptmann, ${ }^{1}$ Oleksandr Burylko, ${ }^{1,2}$ and Peter A. Tass ${ }^{1,3}$ \\ ${ }^{1}$ Institute of Medicine and Virtual Institute of Neuromodulation, Research Centre Jülich, 52425 Jülich, Germany \\ ${ }^{2}$ Institute of Mathematics, National Academy of Sciences of Ukraine, 01601 Kyiv, Ukraine \\ ${ }^{3}$ Department of Stereotaxic and Functional Neurosurgery, University Hospital, 50924 Cologne, Germany
}

(Received 10 November 2006; revised manuscript received 10 April 2007; published 18 June 2007)

\begin{abstract}
We present a simplified phase model for neuronal dynamics with spike timing-dependent plasticity (STDP). For asymmetric, experimentally observed STDP we find multistability: a coexistence of a fully synchronized, a fully desynchronized, and a variety of cluster states in a wide enough range of the parameter space. We show that multistability can occur only for asymmetric STDP, and we study how the coexistence of synchronization and desynchronization and clustering depends on the distribution of the eigenfrequencies. We test the efficacy of the proposed method on the Kuramoto model which is, de facto, one of the sample models for a description of the phase dynamics in neuronal ensembles.
\end{abstract}

DOI: 10.1103/PhysRevE.75.066207

PACS number(s): 05.45.Xt, 87.18.Sn, 87.19.La

\section{INTRODUCTION}

Spike timing-dependent plasticity (STDP) is considered as being a fundamental mechanism for learning and memory in nervous systems $[1,2]$. A synapse conveys signals from a presynaptic to a postsynaptic neuron. Its strength crucially depends on the timing between the actions of the two neurons. It has been shown that if a presynaptic spike advances the postsynaptic spike, the synapse is potentiated (the synaptic weight increases), whereas in the opposite case the synapse is depressed (its weight decreases). The detailed molecular processes underlying STDP remain a matter of debate $[1,2]$.

We here focus on the effects of STDP on macroscopic phenomena, especially on the STDP induced coexistence of distinct patterns of synchronization and connectivity in groups of oscillatory neurons. We choose a phenomenological level of description to reveal fundamental consequences of STDP action. For this, we incorporate STDP [1] into a generic network of phase oscillators, which approximates the dynamics of a network of synaptically interacting neurons (see [3-5]). A model neuron (i.e., phase oscillator) produces an action potential whenever its phase equals $0 \bmod 2 \pi$. We start with the Kuramoto model of coupled phase oscillators $[3,7]$

$$
\dot{\theta}_{i}=\omega_{i}+\frac{1}{N} \sum_{j=1}^{N} K_{i j} g\left(\theta_{i}-\theta_{j}\right), \quad i=1, \ldots, N,
$$

where $\theta_{i}$ are the phases of the individual oscillators (i.e., neurons), $\omega_{i}$ are the natural frequencies (spiking rates), and $K_{i j}$ are the coupling coefficients (synaptic weights). The coupling function reads $g(x)=-\sin x$. The Kuramoto model (1) has been used to study synchronization processes in various fields of the natural sciences (see, e.g., $[3,6]$ ) and in medicine [5].

A recent numerical study in the Kuramoto model with couplings subjected to STDP suggests that in a certain parameter range a desynchronized (weakly coupled) state coexists with a synchronized (strongly coupled) state [8]. We have investigated whether STDP generically leads to a coex- istence of synchronized states and desynchronized states. In particular, we show that in the Kuramoto model STDP gives rise to a multistability of synchronized, desynchronized, and cluster states, provided the STDP learning rule is asymmetric, as observed in experiments.

\section{MODEL}

In the standard $N$-dimensional Kuramoto model without plasticity $\left(K_{i j}=K=\right.$ const $)$, desynchronization occurs when the parameter $K$ decreases below a critical bifurcation value $K_{c}$ : The averaged frequencies $\bar{\omega}_{i}$ of the individual oscillators $\theta_{i}$ split from the common mean frequency $\Omega=\Sigma \omega_{i} / N$. The number of unequal frequencies $\bar{\omega}_{i}$ grows with further decreasing $K$ until at some $K_{d}$ full desynchronization is achieved. A generic property of the standard Kuramoto model without plasticity is an uniqueness of its limiting state (except for atypical narrow parameter regions at the borders between different states [7]).

In order to incorporate the mechanism of plasticity in the Kuramoto model (1), we assume that the elements of the coupling matrix $K_{i j}$ are varied in accordance with the STDP learning rule: Let a presynaptic spike from the $j$ th neuron arrive at the $i$ th neuron at time $t_{\text {pre }}$, whereas a postsynaptic spike is generated in the $i$ th neuron at time $t_{\text {post }}$. The value of $K_{i j}$ increases (synaptic potentiation) or decreases (depression) depending on the sign of the spike-timing difference $\Delta t=t_{\text {post }}-t_{\text {pre }}$. A potentiation occurs when the postsynaptic spike follows the presynaptic spike, i.e., $\Delta t>0$. Conversely, a depression takes place if $\Delta t<0$. As shown experimentally [1], the amount of the change induced in the synaptic weight $\Delta K_{i j}$ decreases exponentially with increasing modulus of the spike-timing difference $\Delta t$ as follows:

$$
\begin{gathered}
\Delta K_{i j}=\varepsilon_{p}\left(\alpha-K_{i j}\right) \exp \left\{-\Delta t / \tau_{p}\right\} \quad(\Delta t>0), \\
\Delta K_{i j}=-\varepsilon_{d} K_{i j} \exp \left\{\Delta t / \tau_{d}\right\} \quad(\Delta t<0) .
\end{gathered}
$$

The multiplier $\alpha-K_{i j}$ in the second relation is added to prevent from an unphysiological growth of the synaptic weights: this implies that all $K_{i j}$ are positive and bounded by 
the maximal coupling strength denoted by $\alpha$. Moreover, potentiation is strong and depression is mild if the synapse is weak (i.e., if $K_{i j}$ is small) and, conversely, potentiation is mild and depression strong if $K_{i j}$ is large and close to $\alpha$.

Going over to phase variables $\theta_{i}$ of Eq. (1) and taking into account the slow variation of $K_{i j}$ with respect to the fast spiking dynamics, it is natural to rewrite the STDP rule in the time-continuous form

$$
\dot{K}_{i j}=\varepsilon \begin{cases}\left(\alpha-K_{i j}\right) \exp \left\{\left(\theta_{i}-\theta_{j}\right) / \tau_{p}\right\}, & \left(\theta_{i}-\theta_{j}\right) \in[-\pi, 0] \\ -K_{i j} \exp \left\{-\left(\theta_{i}-\theta_{j}\right) / \tau_{d}\right\}, & \left(\theta_{i}-\theta_{j}\right) \in[0, \pi],\end{cases}
$$

where, for simplicity, a unique small parameter $\varepsilon$ (instead of two of them, $\varepsilon_{p}$ and $\varepsilon_{d}$ ) is introduced.

The Kuramoto model with synaptic plasticity, Eqs. (1) and (2), is a system of $N^{2}$ ordinary differential equations defined on the Cartesian product of the $N$-dimensional torus $\mathrm{T}^{N}$ (phase variables $\theta_{i}$ ) and the $N(N-1)$-dimensional Euclidean space $\mathbb{R}^{N(N-1)}$ (coupling variables $K_{i j}$ ). The STDP learning rule is asymmetric due to unequal coefficients $\tau_{p}$ and $\tau_{d}$. In experiments [1], the coefficients are estimated as $\tau_{p}$ $\simeq 16.8 \mathrm{~ms}$ and $\tau_{d} \simeq 33.7 \mathrm{~ms}$, and hence, depression is approximately twice more effective than potentiation. In the numerical experiments below we mostly choose $\tau_{p}=0.15$ and $\tau_{d}=0.3$.

It is important that Eqs. (1) and (2), incorporate the main experimental and theoretical findings for the STDP learning rule (see $[1,2]$, and references therein). Indeed, synaptic conductance equations (2) assume that strong synapses undergo relatively less potentiation than weak ones [due to the multiplier $\left(\alpha-K_{i j}\right)$ ], whereas relative depression does not depend on the synaptic strength (given by the multiplier $-K_{i j}$ ). Competition between all synapses of a neuron in the network, and their automatic balance is established by the Kuramoto model (1). Actually, it controls the timing of postsynaptic spikes depending on all presynaptic spikes arriving to the neuron. Prevalence of depression compared to potentiation is determined by different exponents $\tau_{d}$ and $\tau_{p}$, where $\tau_{d}>\tau_{p}$. Below we show that the asymmetry of the STDP learning rule is crucial for the multistability phenomenon. The phenomenon is manifested as coexistence of synchronized, desynchronized, and many other clustered states of different configurations in wide enough regions of the parameters space [12]. (In a Kuramoto model with a Hebbian, cosine synaptic learning rule coexistence of a special type of cluster states was found by [9]. The clusters there are a result of slow-fast motions in the degenerate case $\varepsilon \rightarrow 0$, where a synchronized group of oscillators joins desynchronized oscillators, which are uncoupled).

Before studying models (1) and (2), we note that-as will be shown below-the multistability phenomenon is neither significantly affected by varying the parameter $\varepsilon$ (provided $\varepsilon$ is not unphysiologically large enough; in that case multistability can disappear) nor by approximating the discontinuous functions in Eq. (2) by smooth ones (as proposed, e.g., by [10]). We demonstrate that the main dynamical features of the plasticity model are revealed already in the discontinuous
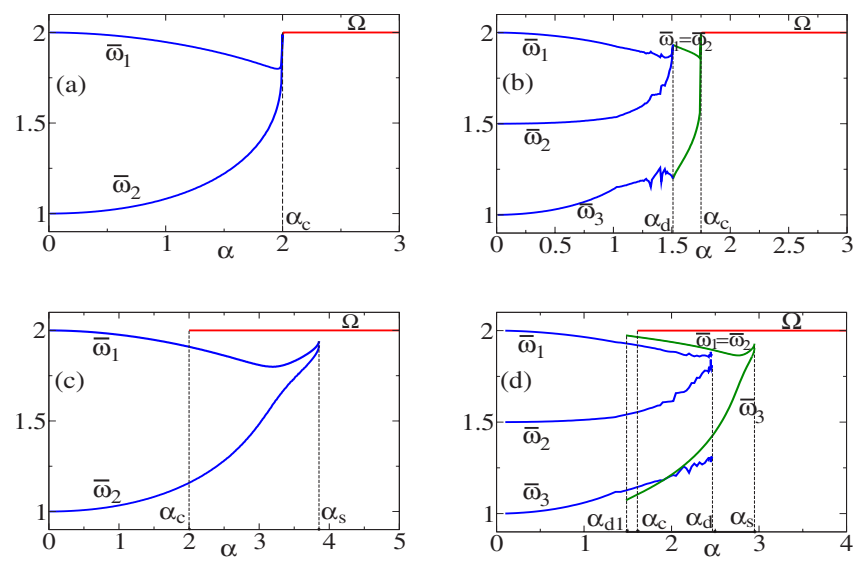

FIG. 1. (Color online) Frequency diagrams for Eqs. (1) and (2) with $N=2[(\mathrm{a}),(\mathrm{c})]$ and $N=3[(\mathrm{~b}),(\mathrm{d})]$, and with an equally spaced distribution of the natural frequencies $\omega_{i}$ in the interval $[1,2]$. (a) and (b): no multistability for a symmetric STDP rule $\left(\tau_{p}=\tau_{d}\right.$ $=0.3$ ); (c) and (d): multistability arises for an asymmetric STDP rule $\left(\tau_{p}=0.15, \tau_{d}=0.3\right)$. Red: fully synchronized state with common frequency $\Omega=\omega_{1}$; blue: fully desynchronized states with different average frequencies $\bar{\omega}_{i}$; green: two-cluster states with $\bar{\omega}_{1}=\bar{\omega}_{2} \neq \bar{\omega}_{3}$. Parameter $\varepsilon=0.5$.

case, which may be considered as the simplest STDP rule, which can further be elaborated.

\section{STDP ASYMMETRY IMPLIES MULTISTABILITY}

In this section we demonstrate that, when introducing plasticity in the Kuramoto model as proposed in Eqs. (1) and (2) the desynchronization transition changes its course from supercritical to subcritical, which happens only if the STDP rule is asymmetric [i.e., $\tau_{d}>\tau_{p}$ in Eqs. (2)]. Figure 1 illustrates a typical course of the desynchronization transition for $N=2$ and $N=3$ oscillators occurring for varying maximal coupling parameter $\alpha$. For $\tau_{d}=\tau_{p}$ there is no mutistability: The desynchronization happens just at $\alpha_{c}$ when the fully synchronized state, given by a common frequency $\Omega=\omega_{1}$, disappears in a saddle-node bifurcation [Figs. 1(a) and 1(b)]. If the STDP rule is asymmetric, i.e., $\tau_{d}>\tau_{p}$, the situation is different: There exists an $\alpha$-parameter interval, namely, for $\alpha$ between $\alpha_{c}$ and $\alpha_{s}$, where the fully synchronized state coexists with a desynchronized state $[N=2$, Fig. $1(\mathrm{c})]$, or coexists with two desynchronized states $[N=3$, Fig. $1(\mathrm{~d})]$ which are a fully desynchronized state of three different average frequencies $\bar{\omega}_{1} \neq \bar{\omega}_{2} \neq \bar{\omega}_{3}$ and a two-cluster state with average frequencies $\bar{\omega}_{1}=\bar{\omega}_{2} \neq \bar{\omega}_{3}$.

In general, we find that the multistability interval $\left(\alpha_{c}, \alpha_{s}\right)$ arises in Eqs. (1) and (2) due to the asymmetry of the STDP rule, i.e., when $\tau_{p}$ becomes smaller than $\tau_{d}$. There is an additional constraint for the existence of a nontrivial multistability interval: The parameter $\varepsilon$ must not be large enough, e.g., in the case in Fig. 1(c) $\varepsilon<\varepsilon_{M} \approx 60.95$ has to be fulfilled). As illustrated in Fig. 4 below, the interval $\left(\alpha_{c}, \alpha_{s}\right)$ expands with decreasing $\tau_{p}$ so that the length of $\left(\alpha_{c}, \alpha_{s}\right)$ grows, probably, infinitely as $\tau_{p}$ tends to zero.

The mechanisms of the phenomenon can be visually illustrated in the simplest case of $N=2$ oscillators. Then, our 

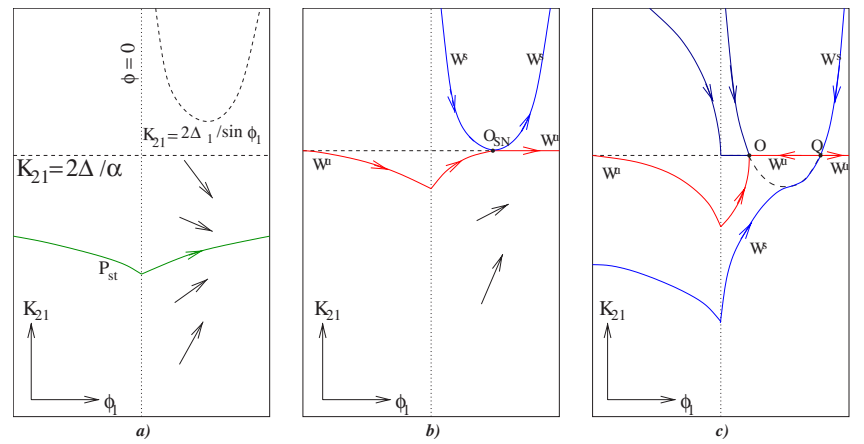

$$
\begin{array}{ll}
\text { Unstable manifold of the saddle } & \mathrm{Q}-\text { - Saddle } \\
\text { Stable manifold of the saddle } & \mathrm{O}-\text { - Stable node } \\
\text { Stable limit cycle } & \mathrm{O}_{\mathrm{SN}}-\text { Saddle--node } \\
& \mathrm{P}_{\mathrm{st}}-- \text { Stable periodic orbit }
\end{array}
$$

FIG. 2. (Color online) Schematic representation of the vector field transformations of the system (3) in the the symmetric case $\tau_{p}=\tau_{d}$, with increasing parameter $\alpha$.

models (1) and (2) contains four equations; its state space is $T^{2} \times R^{2}$, but, the actual dynamics is three-dimensional: instead of two phases $\theta_{1}$ and $\theta_{2}$ one can introduce a phase difference variable $\varphi=\theta_{1}-\theta_{2}$ and then, the model is reduced to the form:

$$
\begin{gathered}
\dot{\varphi}=\Delta_{1}-\frac{K_{12}+K_{21}}{2} \sin \varphi, \\
\dot{K}_{12}=\varepsilon \begin{cases}\left(\alpha-K_{12}\right) \exp \left\{\varphi / \tau_{p}\right\}, & \varphi \in[-\pi, 0] \\
-K_{12} \exp \left\{-\varphi / \tau_{d}\right\}, & \varphi \in[0, \pi],\end{cases} \\
\dot{K}_{21}=\varepsilon \begin{cases}-K_{21} \exp \left\{\varphi / \tau_{d}\right\}, & \varphi \in[-\pi, 0] \\
\left(\alpha-K_{21}\right) \exp \left\{-\varphi / \tau_{p}\right\}, & \varphi \in[0, \pi],\end{cases}
\end{gathered}
$$

where $\Delta_{1}=\omega_{1}-\omega_{2}$. In the variables $\left(\varphi, K_{12}, K_{21}\right)$ the fully synchronized state is given by a stable fixed point $O$ $=\left(\arcsin \left(2 \Delta_{1} / \alpha\right), 0, \alpha\right)$ of Eqs. (3), while the desynchronized regime is described by a stable periodic orbit $P_{s t}$ (see Figs. 2 and 3). The desynchronization transition happens at $\alpha_{c}$ $=2 \Delta_{1}$ and is caused by an annihilation of the stable node $O$ and a saddle $Q=\left(\pi-\arcsin \left(2 \Delta_{1} / \alpha\right), 0, \alpha\right)$ in a saddle-node bifurcation at the point $O_{S N}=(\pi / 2,0, \alpha)$. The course of the transition essentially depends on the equality (inequality) of the parameters $\tau_{d}$ and $\tau_{p}$. In the symmetric case $\tau_{d}=\tau_{p}$, the saddle-node bifurcation at $\alpha_{c}$ is, at the same time, a homoclinic bifurcation for the saddle $Q$. The latter gives birth to a stable periodic orbit $P_{s t}$. It exists for all $\alpha<\alpha_{c}$ and is just an image of the desynchronized state with different average frequencies $\bar{\omega}_{1}$ and $\bar{\omega}_{2}$ of the oscillators $\theta_{1}$ and $\theta_{2}$, respectively. See Fig. 2, where the transformations of the system vector field are shown, when $\alpha$ increases through $\alpha_{c}$.

If $\tau_{d}>\tau_{p}$, the saddle-node bifurcation at $\alpha_{c}$ does not coincide with the homoclinic bifurcation and so, cannot give birth to a periodic orbit. We find that the stable periodic orbit $P_{s t}$ for the model (3) is born in this case in a different, greater coupling parameter value $\alpha_{s}>\alpha_{c}$ as a result of a cycle saddle-node bifurcation. In the bifurcation, $P_{s t}$ is born to-
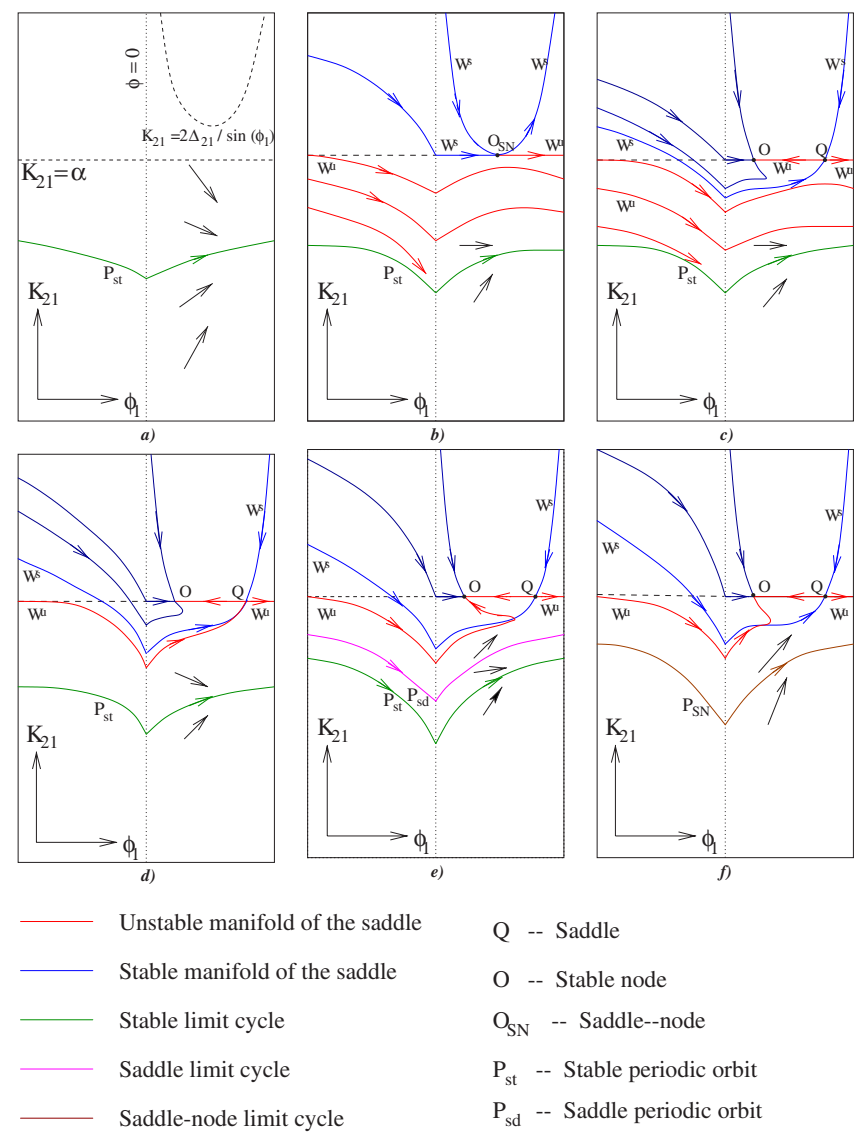

$$
\begin{aligned}
& \mathrm{Q}-\text { - Saddle } \\
& \mathrm{O} \quad \text {-- Stable node } \\
& \mathrm{O}_{\mathrm{SN}} \quad \text {-- Saddle--node } \\
& \mathrm{P}_{\mathrm{st}} \quad-\text { Stable periodic orbit } \\
& \mathrm{P}_{\mathrm{sd}} \text {-- Saddle periodic orbit } \\
& \mathrm{P}_{\mathrm{SN}} \quad-\text { Saddle--node periodic orbit }
\end{aligned}
$$

FIG. 3. (Color online) Schematic representation of the vector field transformations of the system (3) in the asymmetric case $\tau_{p}$ $<\tau_{d}$, with increasing parameter $\alpha$.

gether with a saddle periodic orbit $P_{s d}$. Successive transformations of the vector field are presented in Fig. 3. It is shown that the homoclinic bifurcation of the saddle $Q$ occurs at a parameter value $\alpha_{h}$ which lies in-between that of $\alpha_{c}$ and $\alpha_{s}$, Fig. 3(c). The saddle periodic orbit $P_{s d}$, which is born there, moves in the direction to $P_{s t}$ with increasing $\alpha$, and gets annihilated with $P_{s t}$ in the saddle-node bifurcation at $\alpha_{s}$. The frequencies of the desynchronized states corresponding to $P_{s t}$ are visualized in Fig. 1(c).

Figure 4 demonstrates how the size of the multistability interval $\left(\alpha_{c}, \alpha_{s}\right)$ depends on the asymmetry of the STDP learning rule. In the figure, we fix $\tau_{d}=0.3$ and decrease $\tau_{p}$ from 0.3 (symmetric STDP rule) to zero. Then, the multistability interval $\left(\alpha_{c}, \alpha_{s}\right)$ extends monotonically with decreasing $\tau_{p}$ and, as it is expected, its length grows infinitely as $\tau_{p}$ approaches zero. At the same time, the homoclinic bifurcation curve, denoted by $H$, is bounded and monotonically approaches a limiting value $\alpha_{H}^{0} \approx 2.25$ as $\tau_{p} \rightarrow 0$.

The synchronized state $O$ with common frequency $\Omega$ $=\omega_{1}$ is characterized by a unidirectional coupling scheme such that the faster oscillator $\left(\theta_{1}\right)$ drives the slower one $\left(\theta_{2}\right)$. Indeed, as can be easily concluded from the last two equations of Eqs. (3), in the synchronized, phase-locked regime $K_{12}=0$ and $K_{21}=\alpha$ hold. Also, as one can see, the phase shift between the synchronized oscillators is equal to $\pi / 2$ at $\alpha_{c}$ 


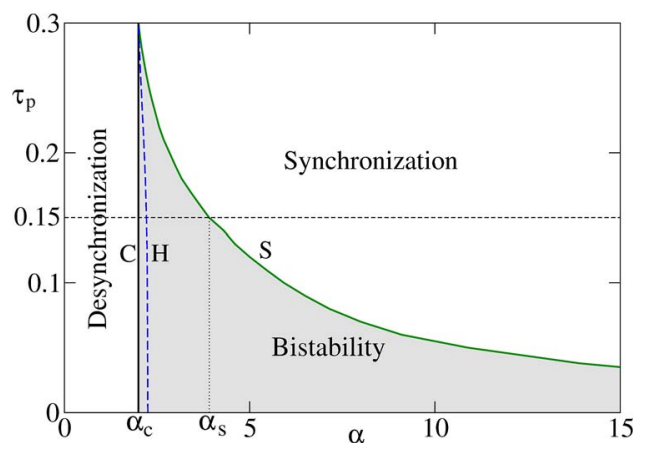

FIG. 4. (Color online) Different dynamical regimes on the parameter plane $\left(\alpha, \tau_{p}\right)$. Parameters $\tau_{d}=0.3, \varepsilon=0.5, \omega_{1}=2$, and $\omega_{1}$ $=1$.

and decreases to 0 with increasing $\alpha \rightarrow \infty$. The order parameter $R$ of the synchronized state can be obtained in this case from the explicit formula

$$
R=\left|\frac{1}{2} \sum_{j=i}^{2} e^{i \theta_{j}}\right|=\frac{1}{2} \sqrt{2\left(1+\sqrt{1-\frac{4 \Delta_{1}^{2}}{\alpha^{2}}}\right)} .
$$

Therefore, $R=\sqrt{2} / 2$ at $\alpha_{c}$ and approaches 1 as $\alpha \rightarrow \infty$.

For the desynchronized state given by the cycle $P_{s t}$ both coupling coefficients $K_{12}$ and $K_{21}$ are nonzero and perform temporal oscillations between 0 and $\alpha$. Moreover, the same holds for the sum of them: $0<K_{12}+K_{12}<\alpha$ provided that $\tau_{d}>\tau_{p}$. (In the symmetric case $\tau_{d}=\tau_{p}$ one gets $K_{12}+K_{12}=\alpha$ ). A typical dynamics of the coupling coefficients $K_{12}(t)$ and $K_{21}(t)$ is plotted in Fig. 5 .

In the case of three globally coupled oscillators $[N=3$ in Eqs. (1) and (2)] the actual system dynamics is eightdimensional and acting on $T^{2} \times \mathbb{R}^{6}$. The bifurcation structure in that case is much more complicated, nevertheless, as illustrated in Figs. 1(b) and 1(d), the course of the desynchronization transition is generally similar to the $N=2$ case. In particular, the fully synchronized phase-locked state $O$ is given by the maximal frequency $\Omega=\omega_{1}$. It disappears with decreasing $\alpha$ in a saddle-node bifurcation at a critical bifurcation value $\alpha_{c}$. [The value of $\alpha_{c}$ is different from the $N=2$ case (see the next chapter)]. Two nonsynchronized states, a desynchronized and a clustered, end in cycle saddle-node

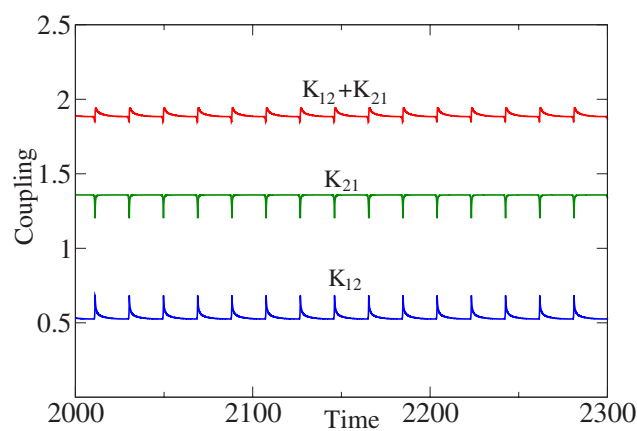

FIG. 5. (Color online) Typical graphs of the variables $K_{12}(t)$ and $K_{21}(t)$, and their sum $K_{12}(t)+K_{21}(t)$. Parameters $\alpha=3.0, \tau_{p}=0.15$, $\tau_{d}=0.3, \varepsilon=0.5, \omega_{1}=2$, and $\omega_{1}=1$. bifurcations at $\alpha_{d}$ and $\alpha_{s}$, respectively. By the analogy with the $N=2$ case, we suggest that saddle periodic orbits which are born in the saddle-node bifurcations last with decreasing $\alpha$ up to respective homoclinic bifurcations, where they cease to exist. We leave "further detailizing" of the $N=3$ bifurcation structure for future study.

\section{SYNCHRONIZED PHASE-LOCKED STATE}

In this section we perform a complete analytical analysis of the fully synchronized state of Eqs. (1) and (2), denoted as $O$. $O$ is given by a unique stable equilibrium of the reduced system in phase differences $\varphi_{i}=\theta_{1}-\theta_{i+1}$ as follows:

$$
\begin{aligned}
\dot{\varphi}_{i}= & \Delta_{i}-\frac{1}{N}\left[\left(K_{1, i+1}+K_{i+1,1}\right) \sin \varphi_{i}\right. \\
& \left.+\sum_{j=1, j \neq i}^{N-1}\left[K_{1, j+1} \sin \varphi_{j}+K_{i+1, j+1} \sin \left(\varphi_{i}-\varphi_{j}\right)\right]\right],
\end{aligned}
$$

where $\Delta_{i}=\omega_{1}-\omega_{i+1}, i=1, \ldots, N-1$. To find $O$, note that in any equilibrium point of Eqs. (4) and (2), each $K_{i j}$ equals either 0 or $\alpha$ [which follows from Eq. (2) due to phase locking of $O]$. Suppose for definiteness, all natural frequencies $\omega_{i}$ be different and ordered as $\omega_{1}>\omega_{2}>\cdots>\omega_{N}$. This implies $K_{i j}=\alpha$ for $i>j$, and $K_{i j}=0$ else. By substituting the values of $K_{i j}$ into Eq. (4), one can get $O$ from the skew-product system

$$
\Delta_{i}-\frac{\alpha}{N}\left[\sin \varphi_{i}+\sum_{l=1}^{i-1} \sin \left(\varphi_{i}-\varphi_{l}\right)\right]=0, \quad i=\overline{1, N-1}
$$

This implies, in particular, that the common frequency $\Omega$ of the synchronized state $O$ is equal to the maximal natural frequency $\omega_{1}$. Indeed, in the synchronized phase-locked state all $K_{1 j}=0$, so that the first equation of Eq. (1) is reduced to $\dot{\theta}_{1}=\omega_{1}$. The other phases $\theta_{i}$ rotate with the same frequency $\omega_{1}$ due to the phase locking. It follows from Eq. (5) that the existence and stability of the state $O$ is defined by relations between the differences of the natural frequencies $\Delta_{i}=\omega_{1}$ $-\omega_{i+1}$ and the parameter $\alpha$, and is not affected by the other parameters $\varepsilon, \tau_{1}$, and $\tau_{2}$ (nevertheless, the latter control the basin of $O$ ). It is important to point out that in the synchronized phase-locked state $O$, the coupling between the oscillators is unidirectional and downwards oriented, i.e., from oscillators with greater to smaller eigenfrequencies.

Summing up the results of this section we formulate the following:

Theorem. There exists a critical coupling parameter value $\alpha_{c}>0$, which depends on the distribution of the natural frequencies $\omega_{1}, \omega_{2}, \ldots, \omega_{N}$, but does not depend on the other parameters $\tau_{1}, \tau_{2}$, and $\varepsilon>0$, such that

(1) for $\alpha \geqslant \alpha_{c}$, the Kuramoto model with synaptic plasticity, Eqs. (1) and (2), has the synchronized phase-locking state $O$ given by the unique stable equilibrium of Eqs. (5). The synchronized state is characterized by an hierarchical unidirectional coupling structure, where the fastest oscillator imposes its frequency on all others;

(2) there is no synchronized phase-locked states for $\alpha$ $<\alpha_{c}$. 


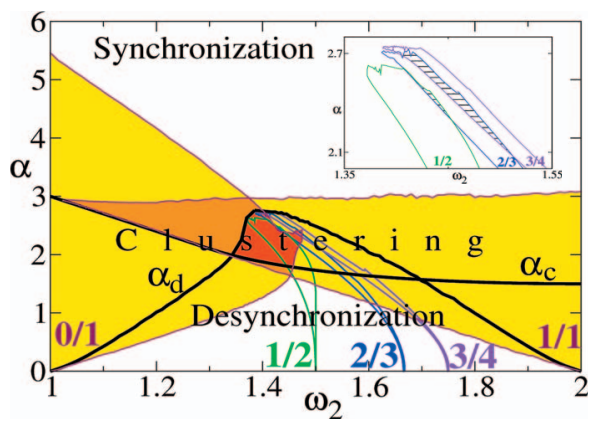

FIG. 6. (Color) Two-parameter multistability diagram for Eqs. (1) and (2) with $N=3$. Horizontal axis: $\omega_{2}$ varies between $\omega_{3}=1$ and $\omega_{1}=2$; vertical axis: $\alpha$. Regions of synchronization (for $\alpha \geqslant \alpha_{c}$ ) and full desynchronization (for $\alpha<\alpha_{d}$ ) are delineated by bold curves $\alpha_{c}$ and $\alpha_{d}$. Two clustering regions given by the resonant tongues $C_{0 / 1}$ and $C_{1 / 1}$ are shown in yellow. They overlap in orange and red regions where three and four different states, respectively, coexist. Overlap of the main resonant tongues $D_{1 / 2}, D_{2 / 3}$, and $D_{3 / 4}$ is illustrated in the inset. Parameters $\tau_{p}=0.15, \tau_{d}=0.3, \varepsilon=0.5$.

From Eq. (5) we obtain in the simplest $N=2$ case $\alpha_{c}$ $=2 \Delta_{1}$ (see Sec. III for details). We have also explicitly found $\alpha_{c}$ for the case of $N=3$ oscillators as follows:

$$
\alpha_{c}=\min \left\{3 \Delta_{1} ; \frac{3 \Delta_{2}^{2}}{2 \sqrt{\Delta_{2}^{2}-\Delta_{1}^{2}}}\right\}
$$

(valid under the condition $\Delta_{2}>\Delta_{1}>0$ ).

\section{MULTISTABILITY VERSUS NATURAL FREQUENCIES}

The simplest nontrivial case, when different desynchronized states coexist is given by three coupled oscillators $[N$ $=3$ in Eqs. (1) and (2)]. In Fig. 6 a two-parameter bifurcation diagram is plotted which shows the multistability dynamics under a variation of the natural frequencies. For this, $\omega_{1}$ and $\omega_{3}$ are fixed, and $\omega_{2}$ is varied. Four different dynamical regimes emerge depending on the parameters $\omega_{2}$ and $\alpha$ : the fully synchronized state $O$ (above the bifurcation curve $\alpha_{c}$ ); the fully desynchronized state $D$ (below the curve $\alpha_{d}$ ); and two 2-cluster states $C_{0 / 1}$ and $C_{1 / 1}$ with average frequencies $\bar{\omega}_{1}=\bar{\omega}_{2} \neq \bar{\omega}_{3}$ and $\bar{\omega}_{1} \neq \bar{\omega}_{2}=\bar{\omega}_{3}$, respectively (in the yellow re-

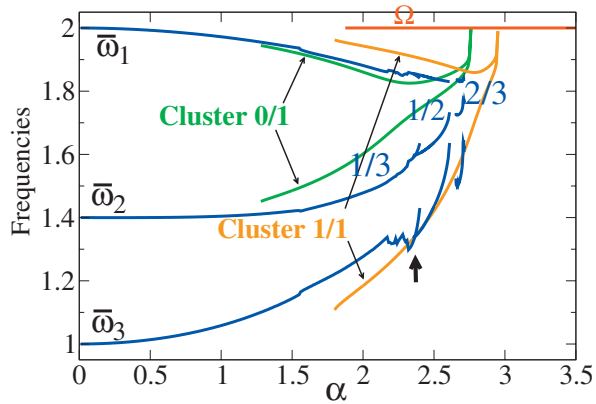

FIG. 7. (Color) Frequency diagram for Eqs. (1) and (2) with $N=3$. $\omega_{2}=1.4$; the other parameters as in Fig. 6. The arrow indicates coexistence of two different fully desynchronized states.

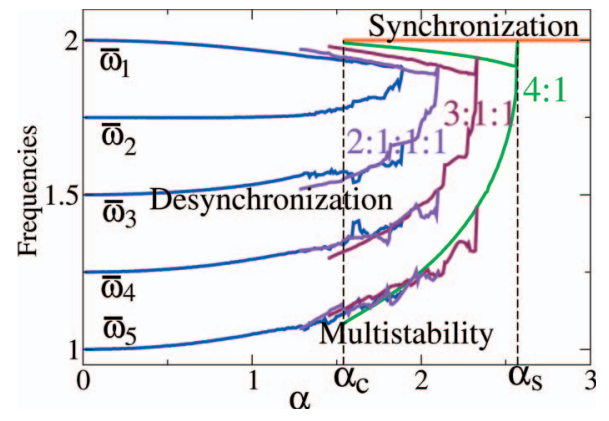

FIG. 8. (Color) Frequency diagram for Eqs. (1) and (2) with $N=5 ; \omega_{i}=2-(i-1) /(N-1), i=1, \ldots, N$. Other parameters as before.

gion; their overlap is shown in orange and red). As one can see, for any fixed $\omega_{2}$ there exists at least one $\alpha$-parameter interval where the synchronous state coexists with the cluster states and with the fully desynchronized state. The "most multistable" parameter region, where all four states coexist is shown in red. In Fig. 7, a one-dimensional frequency diagram for $\omega_{2}=1.4$ is presented, which appears to be much more complicated compared to the diagram with $\omega_{2}=1.5$ [Fig. 1(d)]. $O, C_{0 / 1}, C_{1 / 1}$, and $D$ coexist in the parameter interval $1.9 \ldots \leqslant \alpha \leqslant 2.7 \ldots$ and, in addition, two coexisting fully desynchronized states can be recognized in a narrow parameter interval around $\alpha \approx 2.4$ (indicated by an arrow).

The multistability phenomenon in the models (1) and (2) can be interpreted as an intersection of resonant tongues $D_{p / q}$ originating from rational points at $\alpha=0$, where the dynamics of Eqs. (1) and (2) inside $D_{p / q}$ are characterized by the rotation number $p / q=\left(\bar{\omega}_{2}-\bar{\omega}_{3}\right) /\left(\bar{\omega}_{1}-\bar{\omega}_{3}\right)$. Three main resonant tongues $D_{1 / 2}, D_{2 / 3}$, and $D_{3 / 4}$ are shown in Fig. 6 . Their intersection is illustrated in the inset. Moreover, the stability regions for the two-cluster states $C_{0 / 1}\left(\bar{\omega}_{1}=\bar{\omega}_{2} \neq \bar{\omega}_{3}\right)$ and
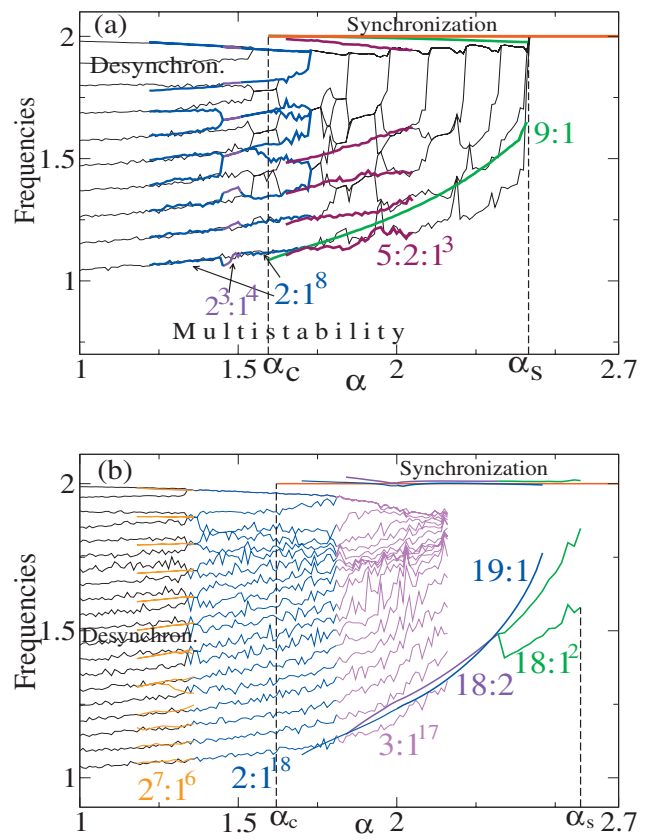

FIG. 9. (Color) Frequency diagram for Eqs. (1) and (2) with (a) $N=10$, (b) $N=20 ; \omega_{i}=2-(i-1) /(N-1), i=1, \ldots, N$. 
$C_{1 / 1}\left(\bar{\omega}_{2}=\bar{\omega}_{3} \neq \bar{\omega}_{1}\right)$ can also be considered as tongues $D_{0 / 1}$ and $D_{1 / 1}$, respectively.

\section{MANY COUPLED OSCILLATORS}

The phenomenon of multistability becomes more developed with an increase of the network size $N$. Figure 8 illustrates a multistable transition from full synchronization to full desynchronization in Eqs. (1) and (2) for $N=5$ oscillators. The desynchronization transition starts at $\alpha_{s}$, where the slowest oscillator $\theta_{5}$ splits from the others, which remain synchronized: a two-cluster state emerges (shown in green) which coexists with the fully synchronized state (red). With further decreasing $\alpha$, the second slowest oscillator $\theta_{4}$ splits, which, in turn, results in a three-cluster state and so on. Therefore, for the $N=5$ case (see Fig. 8), four "standard" desynchronized states occur successively: $4: 1 ; 3: 1: 1 ; 2: 1: 1: 1$; and $1: 1: 1: 1: 1$ (integers denote the number of synchronized oscillators $\phi_{i}$ within a cluster with increasing index $i$ ).

In the network of $N=10$ oscillators, Fig. 9(a), the fully desynchronized state (thin black) ranges from $\alpha=0$ to $\alpha$ $=1.52 \ldots$, where it transforms into a six-cluster state $2^{4}: 1^{2}$. With further increasing $\alpha$, as one can see by a more detailed examination of Fig. 8(b), many other cluster states emerge successively, e.g., $2: 1^{8}, 3^{2}: 2: 1^{2}, 3: 1^{7}, 3: 2^{3}: 1,4^{2}: 1^{2}$, $5: 2: 1^{3}, 6: 2: 1^{2}, 7: 1^{3}, 8: 1^{2}$, and $9: 1$. In the end, the fully synchronized state shows up as a global attractor for $\alpha>\alpha_{s}$. When calculating the bifurcation diagram with decreasing $\alpha$, one can verify that these states exist and are stable at substantially longer parameter intervals compared to the forward calculations. In Fig. 9(a), graphs of the average frequencies for the states $9: 1$ (green), 5:2:13 (maroon), and $2: 1^{8}$ (blue) at their stability intervals are presented. Stability intervals for the other states are also long enough and typically include the critical bifurcation point $\alpha_{c}$ (we do not plot more states for the sake of clarity).

In Fig. 9(b), the multistability phenomenon is illustrated for $N=20$ oscillators. One can observe different cluster states and moreover, the multistability interval $\left(\alpha_{c}, \alpha_{s}\right)$, where the cluster states coexist with full synchronization, appears to be even longer than in the $N=10$ case. In the figure, two groups of clusters can be clearly distinguished: (i) close to synchronization: 20 (full synchronization), 19:1, 18: $1^{2}, 18: 2$, and (ii) close to desynchronization: $1^{20}$ (full desynchronization), $2^{7}: 1^{6}, 2: 1^{18}, 3: 1^{17}$.

Further increasing the number $N$ of the oscillators in the network meets computational difficulties because of fast growing the dimension of Eqs. (1) and (2), which is equal to $N^{2}$. We have made longlasting calculations up to $N=50$ oscillators [then systems (1) and (2) contain 2500 equations] with randomly chosen initial conditions to detect coexistence of different states, and to clarify if the averaged order parameter

$$
R=\lim _{T \rightarrow \infty} \frac{1}{T} \int_{0}^{T}\left|\frac{1}{N} \sum_{j=1}^{N} \exp \left(i \theta_{j}(t)\right)\right| d t
$$

for the desynchronized states (fully desynchronized or clustered) decreases in accordance with the scale law $1 / \sqrt{N}$ as $N$

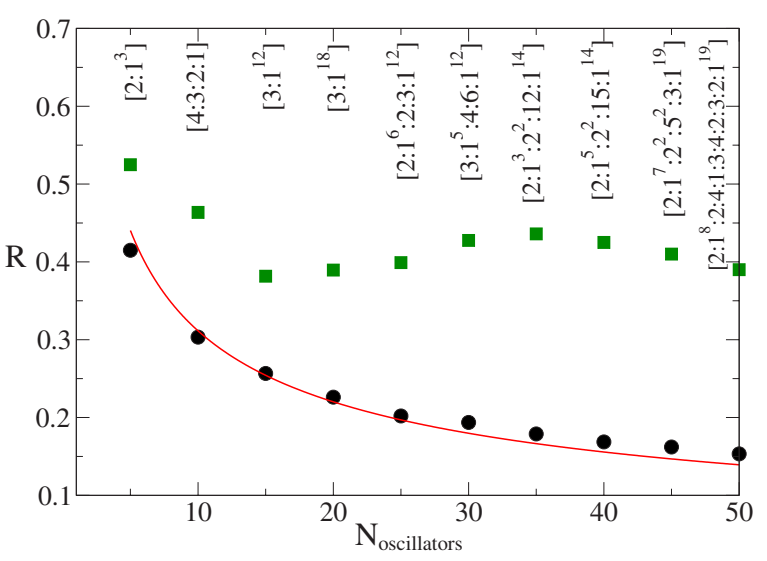

FIG. 10. (Color online) Averaged order parameter $R$ of the desynchronized solutions versus the number of oscillators $N$. Black circles: fully desynchronized solutions for $\alpha=1.0$ and a direct fit $R=C / \sqrt{N}$ by the method of least squares (red); green squares: cluster solutions for $\alpha=1.9$ of the configurations shown above.

increases. As we have found, if the coupling parameter $\alpha$ lies in the desynchronized parameter interval (i.e., approximately $\alpha$ does not exceed the value 1.25) then indeed, $R$ scales in this way. In Fig. 10 perfect agreement with the law is demonstrated for the parameter value $\alpha=1.0$ (black circles). In contrast, in the multistability parameter interval, which is the main subject of our study, there is no such "good" scaling behavior of $R$. Rather (e.g., for $\alpha=1.9$ ), with an increase of $N$ up to $50, R$ saturates at around $0.41 \pm 0.02$ (green squares). The desynchronized solutions are normally not fully desynchronized in this case.

The nondecreasing behavior of the order parameter $R$ in the multistability interval can be explained by the fact that the desynchronized solutions there are not fully desynchronized but clustered only. Indeed, some part of the oscillators in the solutions creates clusters of equal frequencies, other oscillators split off and rotate with different speeds. The number of the oscillators in the clusters can essentially change when varying $N$ and moreover, as one can see in Figs. 7-9, many different cluster states can coexist in the multistability interval. For example, for $N=50$ we found coexistence of the fully synchronized state, when all frequencies are the same and equal to $2.0(R \approx 0.80)$ with two cluster states of the form: $2: 1^{8}: 2: 4: 1: 3: 4: 2: 3: 2: 1^{19}(R \approx 0.39)$ and $2: 1^{20}: 2^{3}: 1: 2: 1^{19}(R \approx 0.48)$. It would be of big interest to clarify what is happening to the order parameter for larger $N>50$, which could be a subject of a future study with a focus on much more time consuming numerics.

To estimate the basin of attraction of different cluster states, we performed calculations of Eqs. (1) and (2) for fixed $\alpha=2.0$ and with randomly chosen initial conditions. The results, which are presented in Fig. 11 in the $N=5$ and $N=20$ cases, show that the size of the basins of attraction of both synchronized and desynchronized regimes, are large enough so that both types of states actually coexist, and therefore, each of these states can be reliably reached by established stimulation protocols $[5,8]$. 

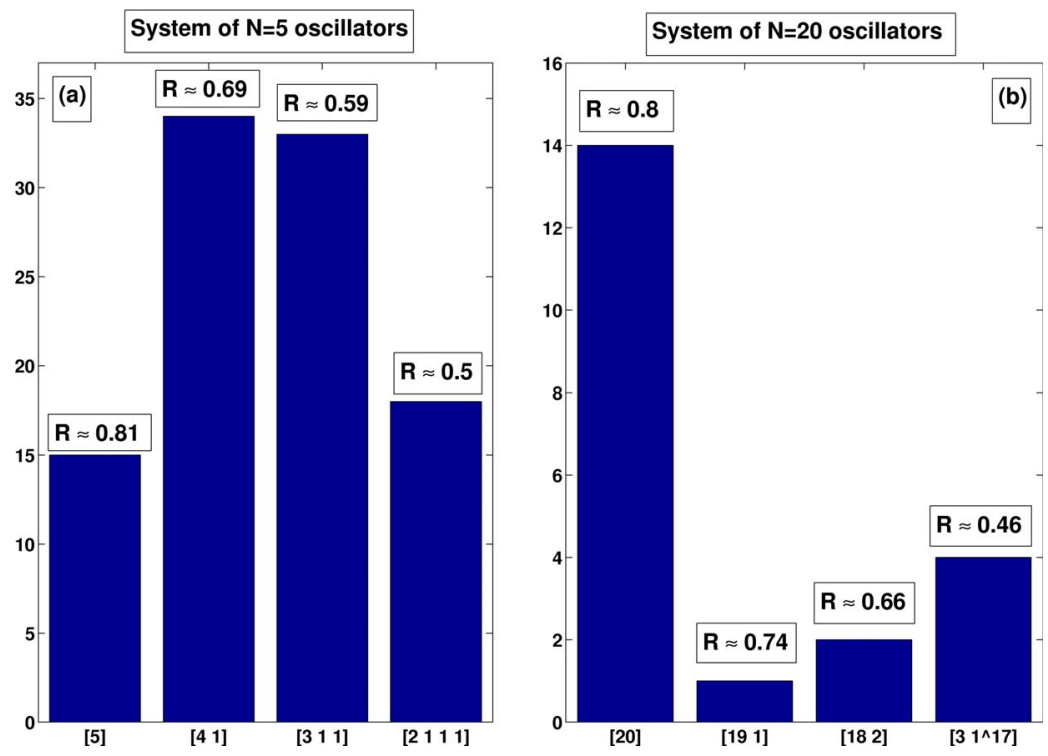

FIG. 11. (Color online) Histograms showing the frequency of different stable states of the model for (a) $N=5$ and (b) $N=20$, where Eqs. (1) and (2) were integrated with randomly chosen initial conditions. Averaged values of the order parameter $R$ for the cluster states are also shown.

\section{CONCLUSION}

In low-dimensional networks of phase oscillators with STDP we have revealed the mechanism leading to multistability. In addition to the results presented here, we found that the multistability becomes more developed by increasing the asymmetry of the STDP rule. Due to STDP, a plethora of coexisting stable cluster states emerges along with the completely synchronized and completely desynchronized states. Intriguingly, a statistical estimation of the basin boundaries of the multitude of attractors reveals that strongly synchronized and strongly desynchronized states are the dominating attractors (Fig. 11). This corresponds to numerical findings in the Kuramoto model with STDP [8] as well as in microscopic neural networks with symmetric STDP [11], both with a large number of oscillators or neurons.

We hypothesize that this low-dimensional mechanism controls also the multistability in the general $N$-dimensional Kuramoto model with STDP, and that the number of cluster states of different configuration, coexisting with full synchronization, grows at least proportionally to $N$. Our results suggest, that long-lasting changes of the network's connectivity can be induced with transient desynchronizing stimuli which shift the system from a stable synchronized state to a stable desynchronized or weakly synchronized state, where the system remains without further intervention. By the same token, periodic stimulations may shift the network from a stable desynchronized state to a stable synchronized state. Theoretical studies indicate that stimulation induced reshaping of neural networks may be relevant for the development of novel brain stimulation techniques (see $[8,11]$ ). In this way it would no longer be necessary to continuously suppress pathological synchronization. Rather, by exploiting STDP, one could achieve long-lasting effects by therapeutically rewiring the target network (see $[8,11]$ ). Of course, the affected brain areas are more complex than a population of phase oscillators. Rather, these brain areas consist of different types of neurons, some being excitatory, others inhibitory. Only a certain percentage of the whole mixed population of neurons appears to be involved in the pathological synchronization processes. Accordingly, our results may serve as a first step performed in a simple model, which can then be extended to a more complex models, containing, e.g., different types of microscopic model neurons.

\section{ACKNOWLEDGMENTS}

We are grateful to L. Tsimring, W. Gerstner, and M. Bazhenov for stimulating discussions. This study was supported by the Network of Excellence in Biosimulation (Grant No. 005137) and by IBM.
[1] H. Markram, J. Lübke, M. Frotscher, and B. Sakmann, Science 275, 213 (1997); G. Q. Bi and M. Poo, J. Neurosci. 18, 10464 (1998); Annu. Rev. Neurosci. 24, 139 (2001); G. M. Wittenberg and S. S.-H. Wang, J. Neurosci. 26, 6610 (2006); J.-P. Pfister and W. Gerstner, ibid. 26, 9673 (2006).

[2] S. Song, K. D. Miller, andL. F. Abbott, Nat. Neurosci. 3, 919 (2000); M. van Rossum and G. Turrigiano, Neurocomputing 38-40, 409 (2001).

[3] Y. Kuramoto, Chemical Oscillations, Waves and Turbulence
(Springer-Verlag, Berlin, 1984); S. H. Strogatz, Physica D 143, 1 (2000).

[4] D. Hansel, G. Mato, and C. Meunier, Europhys. Lett. 23, 367 (1993).

[5] P. A. Tass, Phase Resetting in Medicine and Biology (Springer, Berlin, 1999); Europhys. Lett. 55, 171 (2001); 59, 199 (2002); Phys. Rev. E 66, 036226 (2002); 67, 051902 (2003); Biol. Cybern. 89, 81 (2003).

[6] G. Kozyreff, A. G. Vladimirov, and P. Mandel, Phys. Rev. 
Lett. 85, 3809 (2000); Y. B. Kazanovich and R. M. Borisyuk, Neural Networks 12, 149 (1999); L. S. Tsimring N. F. Rulkov, M. L. Larsen, and M. Gabbay, Phys. Rev. Lett. 95, 014101 (2005); H. Kori and Y. Kuramoto, Phys. Rev. E 63, 046214 (2001); D. Pazó, ibid. 72, 046211 (2005).

[7] Y. Maistrenko, O. Popovych, O. Burylko, and P. A. Tass, Phys. Rev. Lett. 93, 084102 (2004); O. V. Popovych, Yu. L. Maistrenko, and P. A. Tass, Phys. Rev. E 71, 065201(R) (2005).

[8] P. A. Tass and M. Majtanik, Biol. Cybern. 94, 58 (2006).

[9] P. Seliger, S. C. Young, and L. S. Tsimring, Phys. Rev. E 65, 041906 (2002).

[10] H. D. I. Abarbanel, R. Huerta, and M. I. Rabinovich, Proc.
Natl. Acad. Sci. U.S.A. 99, 10132 (2002).

[11] P. A. Tass and C. Hauptmann, 9, 298 (2006); C. Hauptmann and P. A. Tass, BioSystems 89, 173 (2007); P. A. Tass and C. Hauptmann, Int. J. Psychophysiol 64, 53 (2007).

[12] By synchronized (clustered) state we mean a state where all oscillators (at least one group of oscillators) are characterized by the same average frequency, i.e., are phase locked. In the model considered, Eqs. (1) and (2), the synchronized oscillators are normally phase shifted with respect to each other, or they can oscillate with respect to each other with bounded phase difference. Fully desynchronized state means a state with all average frequencies being different. 